\title{
3D MICRO-MACRO FLUID-STRUCTURE MODEL OF PRESSURE RELIEF VALVE LEAK TIGHTNESS
}

\author{
Ali A. Anwar* \\ WEIR Advanced Research Centre \\ Mechanical and Aerospace Engineering \\ University of Strathclyde \\ Technology and Innovation Centre \\ 99 George St, Glasgow, G1 1RD \\ ali.anwar@strath.ac.uk
}

\author{
William Dempster \\ Yevgen Gorash \\ Mechanical and Aerospace Engineering \\ University of Strathclyde, \\ James Weir Building \\ 75 Montrose St, Glasgow, G1 1XJ \\ william.dempster@strath.ac.uk \\ yevgen.gorash@strath.ac.uk,
}

\section{ABSTRACT}

Controlling and assessing the leak tightness of a Pressure Relief Valve (PRV) has been a challenge since the original design of the product. With more stringent demands from the nuclear power industry for leakproof PRV's, closer to the set point, there has been a drive by both industry and academia for a better design method for many known metal-to-metal contacting seal/surface problems. This paper outlines a numerical modelling strategy drawn from industry experience and metrology measurements and investigates the effects of lapping and surface finish on leakage rate. Key influencing parameters of surface form, waviness and roughness are incorporated in the analysis. The numerical approach requires efficient coupling of a non-linear structural Finite Element Analysis (FEA) with a Computational Fluid Dynamic (CFD) solver. This allows the examination of the relationship between deformation of the contacting surfaces, based on the applied spring force, and the resulting micro-flow of gas through any available gaps and the overall leakage to be found. The API527 Seat Tightness methodology is followed to allow leakage rates to be measured and the computational model to be preliminarily validated. Using this model, engineers can adjust and optimise the design of pressure relief valves to find the minimal leakage condition for a given configuration. In addition, the numerical approach can potentially be applied to other metal-to-metal contacting surface components, such as flanges with metal gaskets, and help eliminate leakage.

*Address all correspondence to this author.

\section{INTRODUCTION}

The role a Pressure Relief Valve (PRV) plays in a pressurised system is simple, yet from a safety perspective, arguably the most important. PRV's are commonly used in nuclear power plants for both the primary and secondary reactor coolant systems. The role it plays is to relieve the system of over-pressure; maintaining the pressurised system at a safe level.

A PRV's design has not changed very much since its origin. A known issue with metal-to-metal seal PRV's is leakage which subsequently can cause set-pressure $\left(P_{\text {set }}\right)$ drift. This issue can have a detrimental widespread outcome such as the incident which occurred in 2013 with the US Pilgrim Nuclear Power station. It was reported that 3 of the 4 Pilot Operated PRV's were leaking in the primary coolant cycle which forced the power station to shut-down [1].

Japan's Atomic Energy Agency (JAEA) reviewed the trend of incidents of US power stations (years 2000 to 2006), finding the second dominant cause of set-pressure drift of PRV's being leakage [2].

PRV leak tightness guarantee provided by both manufacturers and consumers is of great importance. A consumer would want to ensure the leak tightness is guaranteed to safeguard working conditions. Manufacturers guarantee leak tightness by complying with standards such as API 527:Seat Tightness of Pressure Relief Valves [3] and dependant on region the equivalent standard would be used i.e. British Standard-BS EN ISO 4126-1:2013 [4]; ASME Standard-ASME PTC 25-2014 [5], etc. When in operation, as a PRV reaches its set-pressure the leakage 
rate increases. Therefore, having the ability to design according to standards and reduce the leakage of a PRV allows valve manufacturers to create a market competitive product.

In the past, organisations such as the Midwest Research Institute (on behalf of NASA) [6] and the European Commission Community Research [7] attempted to understand: why valve leakage occurs; the underlying physics to predict leakage; and monitoring/assessing leakage. To date overall research directly relating to metal-to-metal contact PRV leakage is scarce.

Nonetheless, understanding of PRV leakage can be drawn from relevant fields such as: metal-to-metal contact and gasket seals. When these metal surfaces come into contact in parallel to each other a finite gap or path is present which is dictated by key metrology surface finish quality such as: form; waviness; and roughness. Subsequently, if there is a driving internal pressure the fluid can navigate through the path and exit the valve resulting in leakage [8].

In this paper, a new numerical modelling method is presented to model metal-to-metal contact leakage of PRV's. The metal-to-metal contact surfaces of a PRV are the 'Seat' and 'Disc' (see Fig. 1). This method relies upon measuring the key metrology characteristics of both these 'poli-lapped' surfaces. The surface finish characteristics are integrated into 2 separate geometric models: (1) A full micro-macro model of the PRV contact representing the surface form and waviness; and (2) a partial surface scan representing the roughness. Both these geometric models are numerically analysed using Finite Element Analysis (FEA) to understand the effect on the finite gap due to the spring force. The resultant gap is exported from the micro-macro model and assuming laminar gas micro-flow (ranging from the slip flow to the continuum flow regime) a Computational Fluid Dynamics (CFD) solver is used to find the leakage attributed to the form and waviness.

The PRV of interest for this research is spring-loaded with a $19.25 \mathrm{~mm}$ internal radius. Using the new numerical modelling method, leakage of the Seat-Disc contact up to a set-pressure of $0.5 \mathrm{MPa}$ are presented. Initial validation of the results using a theoretical equation and experimental measurements for the spring-loaded PRV are then discussed.

The numerical modelling tool is then used to investigate Seat length optimisation and how it affects leakage of a PRV. Discussions regarding to the extension of this work and conclusions follow.

\section{VALVE LEAK TIGHTNESS METHODOLOGY}

Within a PRV, a compressed spring applies a force on the disc which is transferred to the seat due to the metal-to-metal contact. As the internal pressure $\left(P_{i n}\right)$ of the fluid increases, the overall force on the disc and seat decreases, until eventually the pressure reaches $\left(P_{\text {set }}\right)$ exceeding the force of the spring, pushing the Disc off of the Seat and opening the valve. When the Disc and Seat are in contact, there is leakage of fluid between the Seat and Disc. Therefore, an investigation of the metrology characteristics of the contacting surfaces is required to determine: the finite gap this creates between the Seat and Disc; how the spring

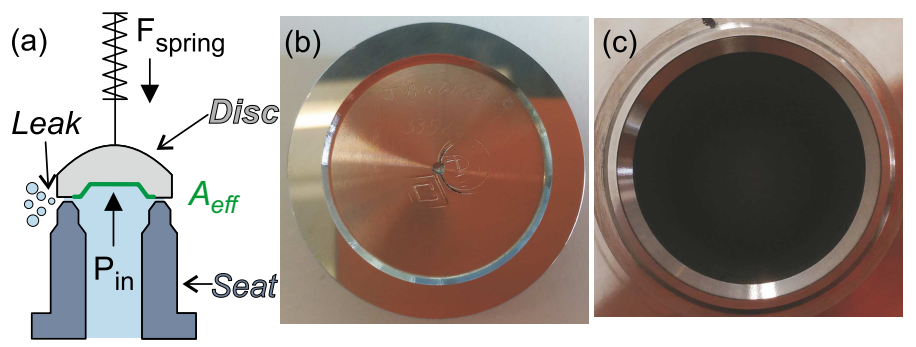

FIG. 1 (a) SIMPLE DIAGRAM OF PRV WITH SPRING FORCE $\left(F_{\text {spring }}\right)$, INTERNAL PRESSURE $\left(P_{i n}\right)$, SEAT, DISC AND EFFECTIVE DISC AREA $\left(A_{e f f}\right)$ HIGHLIGHTED (b) DISC (BOTTOM VIEW) (c) SEAT (TOP VIEW)

force $\left(F_{\text {spring }}\right)$ deforms the finite gap; and ultimately how much fluid passes through the gap.

This numerical method is split up into 4 sections: Metrology; Computer Aided Design (CAD); FEA; and CFD. By finding first the metrology characteristics of the contacting surfaces, $2 \mathrm{CAD}$ models can be created and independently analysed to find the effect of the spring force on the metallic contact surfaces and subsequently the finite gap. The deformed gap between the contacting surfaces in the FEA analysis is exported into a CFD solver allowing analysis of the fluid flow through the gap finding the leakage. Each of these sections is completed in sequence and requires analysis of 2 independent geometric models: (1) a macro-micro model of the seat which incorporates the combined surface form and waviness of both the seat and disc; and (2) a local $100 \mu \mathrm{m}$ by $100 \mu \mathrm{m}$ actual scan converted to a CAD model representing the surface roughness. The local model is used to only advice the first model of the extent of roughness contact. A breakdown of the sections and the two models is represented in a flow diagram in Fig. 2 .

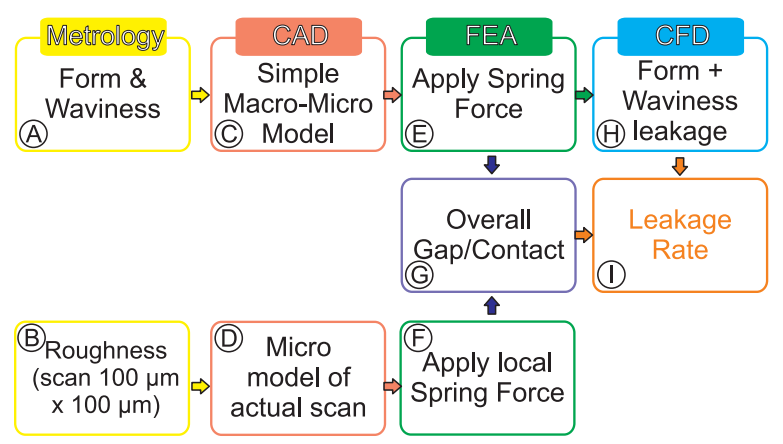

FIG. 2 VALVE LEAK TIGHTNESS METHODOLOGY FLOW DIAGRAM

\section{Metrology}

As mentioned, when the disc and seat are in contact, there is a leakage of fluid between the seat and disc. This leakage is 
associated with a finite gap between the seat and disc contacting surfaces. In turn, the finite gap is a formation of the surface finish quality of the seat-disc contact. Both the seat and disc contact surfaces are 'poli-lapped' i.e. polished and lapped simultaneously, to produce a mirror finish appearance (see Fig. 1), giving the appearance that the surface is 'smooth' or 'flat'; closer examination reveals otherwise.

The key metrology features which require examination to understand the extent of the gap are: average surface form; average waviness $\left(W_{a}, W_{s m}\right)$; and average roughness $\left(R_{a}\right)$. What and how these surface features are interlinked is displayed in Fig. 3 . which shows the roughness being a sub-feature of the waviness, and the waviness being a sub-feature of the form.

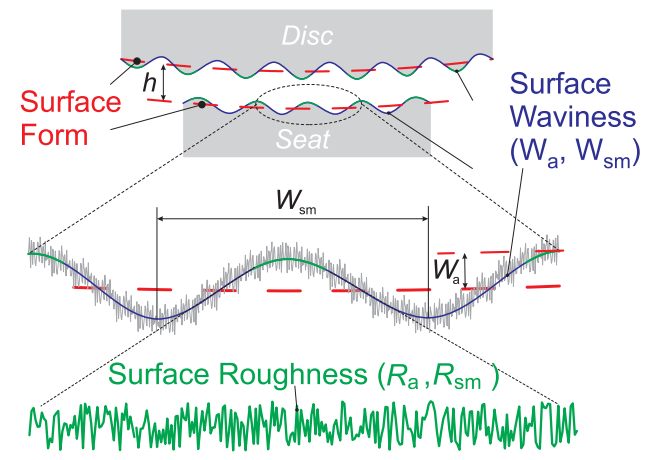

FIG. 3 METROLOGY CHARACTERISTICS: AVERAGE SURFACE FORM; AVERAGE WAVINESS; AND ROUGHNESS

Using a non-contact optical interferometry device the surfaces are examined more closely to reveal the true surface finish quality. The interferometry device used for this paper was an Alicona InfiniteFocus. The average surface form is presented in Fig. 4 with the waviness and roughness characteristics in Table 1

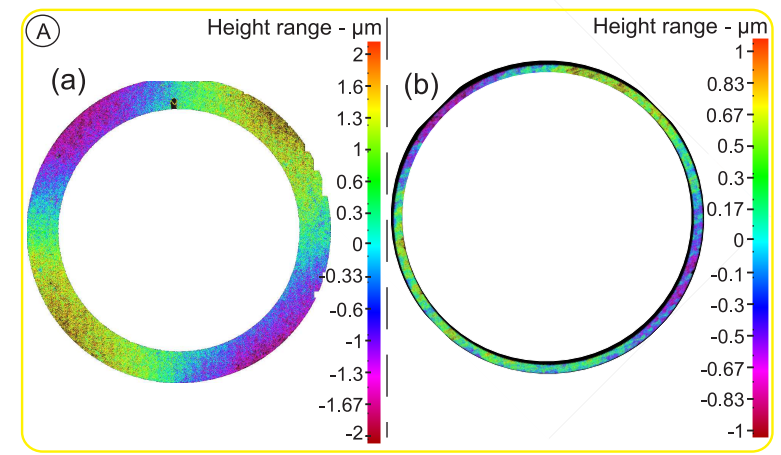

FIG. 4 AVERAGE SURFACE FORM MEASUREMENT OF (a) DISC AND (b) SEAT SCANNED USING AN ALICONA INFINITEFOCUS AT 20X OBJECTIVE MAGNIFICATION

\begin{tabular}{|c|c|c|c|}
\hline & $\begin{array}{c}\text { Mean Form deviation } \\
\text { (Flatness })(\mu \mathrm{m})\end{array}$ & $\begin{array}{c}W_{a}\left(W_{s m}\right) \\
(\mu \mathrm{m})\end{array}$ & $\begin{array}{c}R_{a} \\
(\mu \mathrm{m})\end{array}$ \\
\hline Disc & 4.38 & $0.21(1751)$ & 0.041 \\
\hline Seat & 2.1 & $0.11(2449)$ & 0.068 \\
\hline
\end{tabular}

TABLE 1 Average surface form deviation (flatness), Average Waviness $\left(W_{a}\right)$, Average Waviness spacing $\left(W_{s m}\right)$ and Summing technique parameters. Measured using an Alicona Infinitefocus

From Fig. 4 both the seat and disc show a distinct sinusoidal shape with 2 peaks and 2 troughs. The peaks and troughs for both the disc and seat have a total deviation (flatness) of $4.38 \mu \mathrm{m}$ and $2.1 \mu \mathrm{m}$ respectively. As is expected for a 'poli-lapping' finish quality, the procedure produces a low surface roughness value and the waviness is also very well controlled.

What is apparent is that the surface form, will produce the greatest gap when both surfaces are in contact (irrespective of the orientation of the contacting faces), therefore the greatest contributor to leakage. While the roughness will contribute the least leakage since it would generate the smallest gap between the contacting faces.

Examining the roughness more closely (Fig. 57), the random nature of the surface for a $100 \mu \mathrm{m}$ square sample becomes apparent. Considering the aim of this work is to model leakage, then creating a geometric model of the whole surface of the seat and disc with form, waviness and roughness embedded into one, is not computationally feasible. The reason why a $100 \mu \mathrm{m}$ square area is chosen for this study is to try and reduce any interference of the characteristics of waviness i.e. $100 \mu \mathrm{m} \ll W_{s m}$.

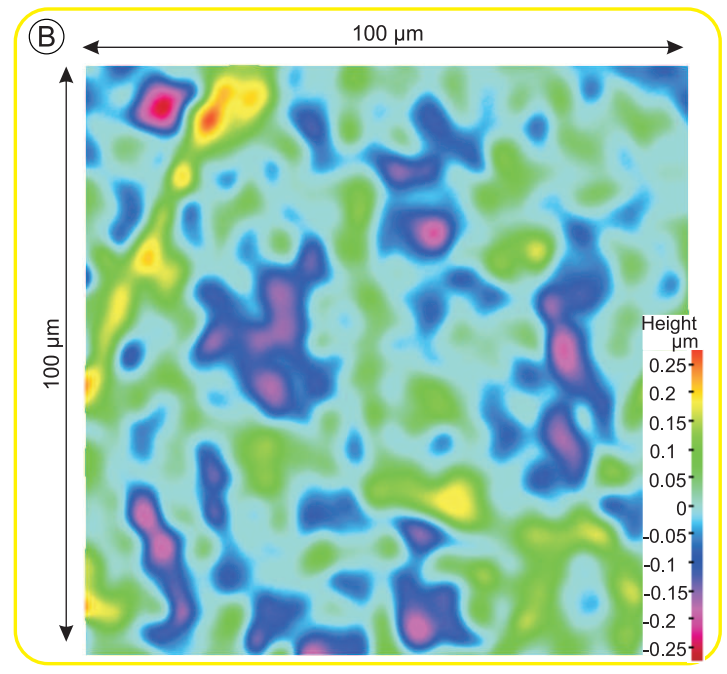

FIG. $5100 \mu \mathrm{m}$ BY $100 \mu \mathrm{m}$ ACTUAL SCAN OF DISC USING THE ALICONA INFINITEFOCUS AT 50X OBJECTIVE MAGNIFICATION 


\section{CAD}

To represent these surface finish features geometrically, but still capture the fluid-flow across all 3 of these key surface characteristics, 2 geometric models are created:

1. Form \& Waviness - a micro-macro model representing the surface form and waviness;

2. Roughness - actual scan (Fig. 5) converted to a CAD model representing the surface roughness.

Form \& Waviness model Both the contact surfaces are geometrically modelled using the summing technique. This technique allows the direct analysis of two contacting surfaces represented into a single equivalent surface in contact with a rigid perfectly flat surface (refer to references [9] and [10]).

Since the average surface form profile follows a sinusoidal shape for both the seat and disc, it can be added together to create the equivalent average surface form profile. This can be created directly using splines driven by an equation in CAD software.

Following the summing technique, the waviness amplitudes $W_{a}$ for the seat and disc would be added together, whilst the waviness spacing $W_{s m}$ is averaged. The waviness for this particular case is modelled using pyramids such as that shown in (Fig. 6). Other methods of representation, such as the fractal based, or actual scans could be utilised to present the waviness which would be more realistic. However, considering the waviness amplitude is about 20 times less than the average surface form for this case, the waviness would contribute much less leakage. Also more realistic surface finish conditions would have a much greater computational cost associated with it.

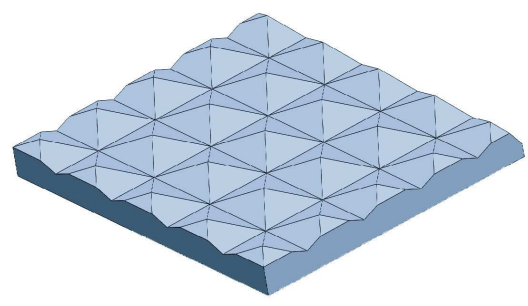

FIG. 6 SIMPLE GEOMETRY OF PYRAMIDS REPRESENTING WAVINESS BASED ON $W_{a}$ AND $W_{s m}$

Using the summing technique and simplistic geometry, the form and waviness surface finish is incorporated into the top of the valve seat as shown in Fig. 7 The average surface form represents a $\frac{1}{4}$ symmetric sinusoidal shape for both the seat and disc. In reality, both these contacting surfaces could rotate around one another, which would change the gap dimensions. For this analysis, we are looking to find the maximum leakage, therefore to facilitate this, based on the rotation of the seat and disc, the peaks of each surface would have to align. However, if the interest was to find the least leakage, the seat and disc would have to be rotated so that the peaks and troughs of each surface would align.

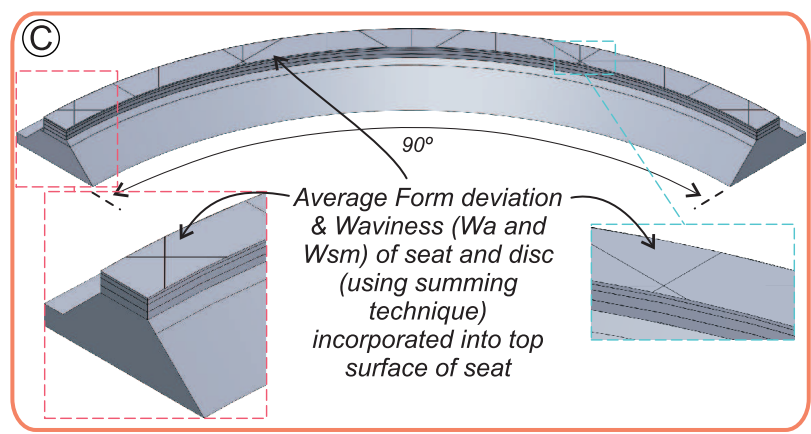

FIG. 7 GEOMETRIC MODEL OF $\frac{1}{4}$ SYMMETRIC VALVE SEAT WITH FORM AND WAVINESS INCORPORATED

Roughness model Since the roughness of the seat and disc is of the lowest magnitude of the 3 metrology parameters, it is the most challenging to incorporate into the Form \& Waviness model for the seat (especially considering meshing capabilities using FEA). Instead a representative scaled model is used to find the average gap size based on the spring force as shown in Fig. 8

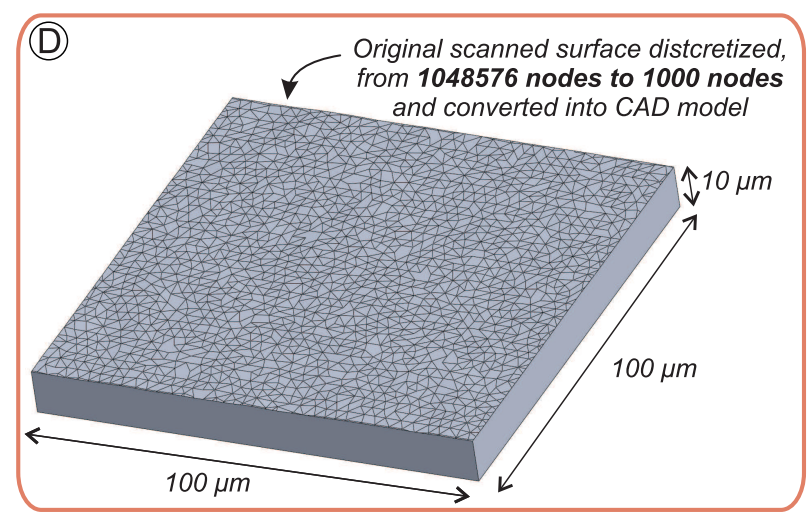

FIG. $8100 \mu \mathrm{m}$ BY $100 \mu \mathrm{m}$ ACTUAL SCAN OF DISC CONVERTED INTO CAD FORMAT

The original scan (Fig. 5) generates 1,048,576 nodes which if directly meshed in FEA or CFD would be computationally challenging and expensive. The Alicona InfiniteFocus, works by measuring a specific area and then traversing to the adjacent area, until the full area of interest has been scanned. It then stitches all this data together generating a surface like that shown in Fig. 5 This method allows a high resolution of the surface to be generated as an 'STL' file, however generating a lot of data. Instead the scan is discretized from 1,048,576 nodes to 1,000 nodes using 2 algorithms: the Poisson-disk distribution [11]; and the ball-pivoting algorithm [12]. Using these algorithms the discretisation yields an $R_{a}$ value less than $5 \%$ of the original scan. Both these algorithms can be found in freeware software such as MeshLab. This discretisation method would also be applicable to other optical-interferometry device measurements. 


\section{FEA}

Using ANSYS ${ }^{\circledR}$ workbench (version 17), the geometric models are analysed using FEA to find the deformation of the contact surfaces due to the spring force in an elastic perfectlyplastic manner.

The disc is made of a Stellite Alloy while the seat is made of AISI $316 \mathrm{~N}(\mathrm{~L})$ steel. Since the summing technique is being utilised, the perfectly flat surface is a rigid surface (SURF154 elements). Since the seat has the equivalent surface finish of both the seat and disc the elastic modulus is calculated using a material joint resistance using Eqn. (1) for both the seat and disc.

$$
\frac{1}{E_{q}}=\left(\frac{1-v_{1}^{2}}{E_{1}}+\frac{1-v_{2}^{2}}{E_{2}}\right) \cdot \frac{1}{2}
$$

The yield stress $\left(\sigma_{y}\right)$ is based on the softer of the two materials in contact, i.e. in this case being the seat.

The boundary conditions for both geometric models are displayed in Fig. 9 and Fig. 10, Both models of the seat are predominantly made up of SOLID187 10-node elements, with the seat and disc contact surfaces associated with CONTA174 8-node and TARGE170 4-node quadratic elements associated with the rigid surface.

The Form \& Waviness FEA model is made up of 108,698 elements and 379,254 nodes. The Roughness model is made up of 1,019,763 elements and 667,268 nodes. The mesh for the contact regions requires a high resolution to capture the micro scale contact deformation as shown in both the figures 9 and 10

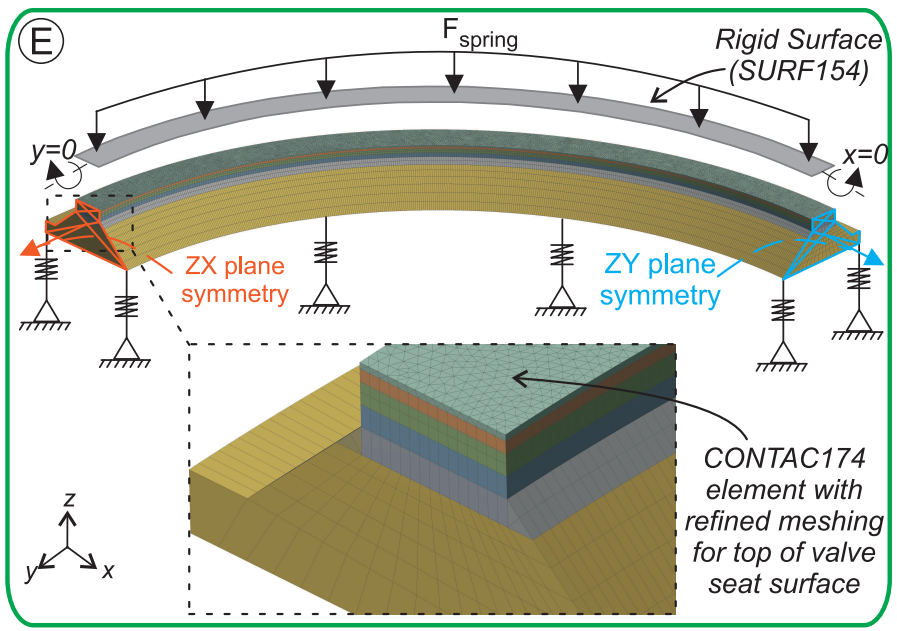

FIG. 9 FE MODEL OF $\frac{1}{4}$ SYMMETRIC VALVE SEAT WITH BOUNDARY CONDITIONS

Only a $\frac{1}{4}$ of the CAD seat is modelled to allow symmetric boundary conditions to be applied in the ZX and ZY planes.

Similarly, the same symmetric boundary planes are applied around the Roughness model to artificially mimic the whole surface.
The bottom of the models have elastic support boundary conditions. This allows geometric simplification of the models while still allowing accurate simulation of the whole geometry.

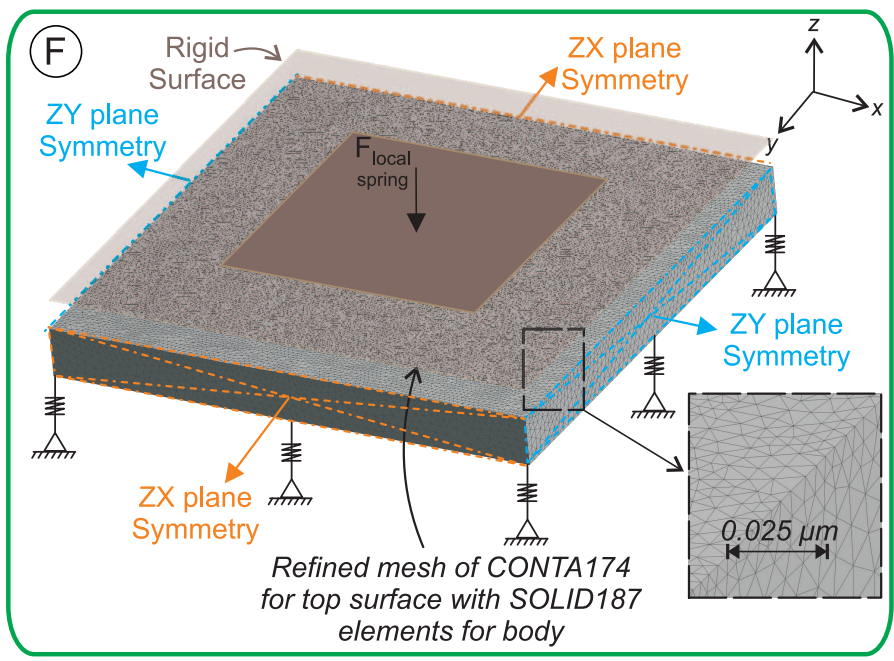

FIG. 10 ROUGHNESS FE MODEL WITH BOUNDARY CONDITIONS

As recommended in API 527 [3], when testing for leakage the spring force is increased to an amount which is capable of withstanding the $P_{\text {set }}$. The internal pressure $\left(P_{i n}\right)$ is then increased up to $90 \%$ of the $P_{\text {set }}$, then the leakage is measured. So to represent this in the FEA, the spring force $\left(F_{\text {spring }}\right)$ is applied to the rigid perfectly flat surface over 2 load steps (Fig. 111) using a static analysis. In the first load step the spring force is increased to a force representative of acting against $P_{\text {set }}$, which for this study is $F_{\text {spring }}=P_{\text {set }} . A_{\text {eff }}$. In the second load step the force is linearly reduced to $10 \%$ of the full spring force. Rather than modelling the $P_{i n}$ as a boundary condition, the internal pressure can be calculated using $P_{\text {in }}=F_{\text {spring }} / A_{\text {eff }}$, allowing artificial representation of the internal pressure up to the $P_{\text {set }}$, mimicking the behaviour which would be seen in reality.

For the Roughness model, the local spring force $\left(F_{\text {local spring }}\right)$ in Fig. 10 is simply the fractional spring force based on the total seat and rigid surface contact area.

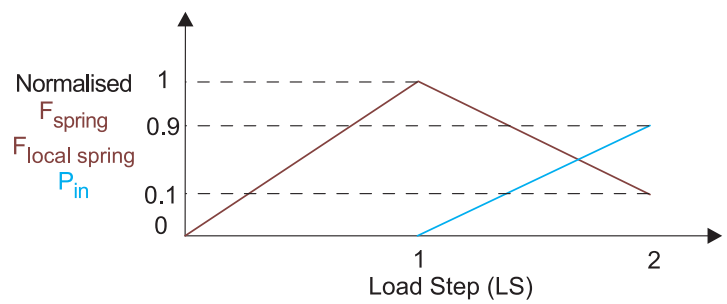

FIG. 11 SPRING FORCE APPLICATION WITH RESPECT TO LOAD STEP 


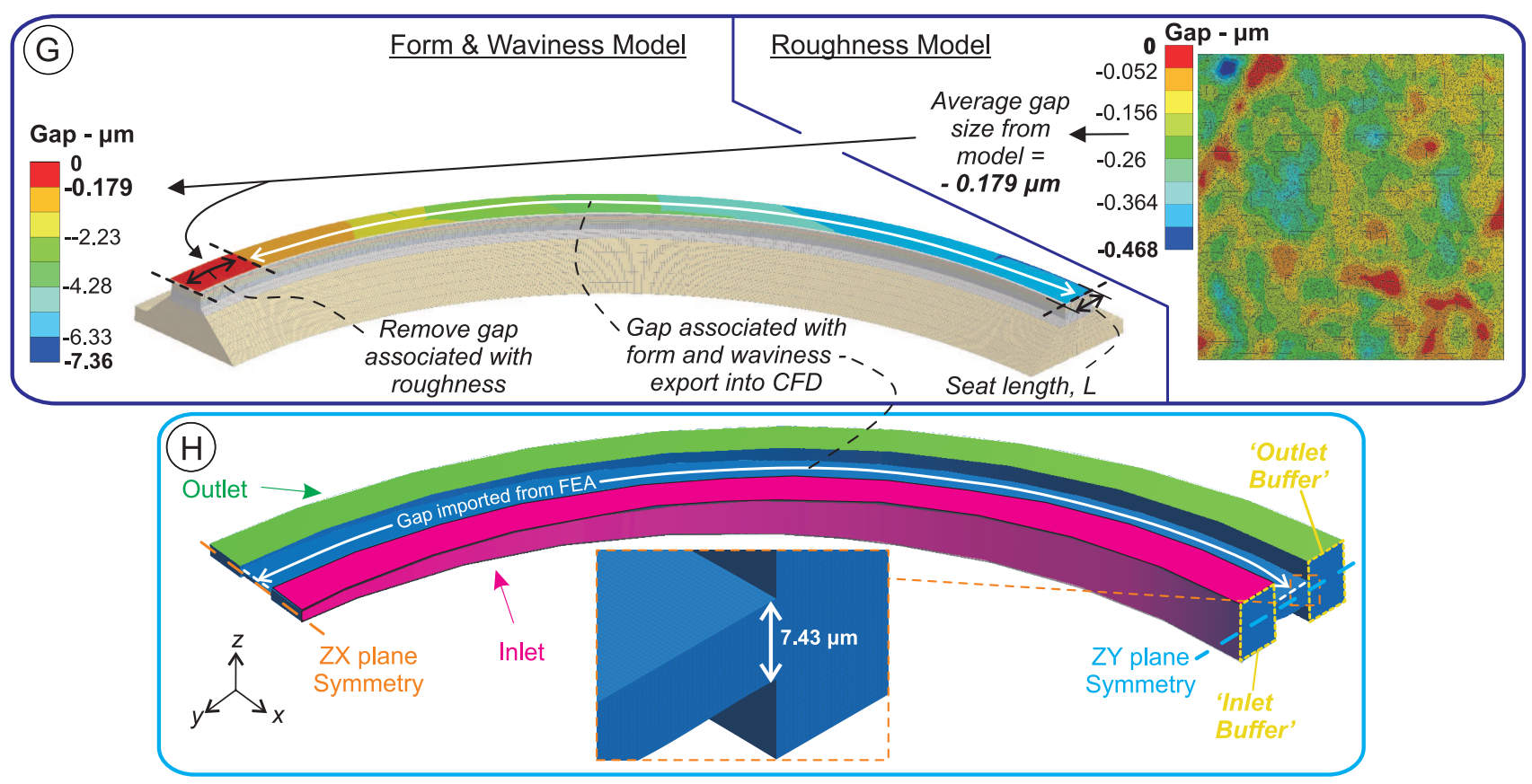

FIG. $12 \mathrm{G}$-OVERALL CONTACT GAP AT $P_{\text {int }} 90 \%$ OF $P_{\text {set }}=0.5 \mathrm{MPA}$ AND H - CFD MODEL OF FORM \& WAVINESS MODEL

Overall contact The FEA models are solved and at the $P_{\text {in }}$ of interest, the deformed gap between the seat and rigid surface needs to be exported as a CAD file from the second load step.

The roughness model is solved at the $P_{i n}$ of interest as well and the average gap size is found. This average gap size is associated to the deformed void space between the rigid surface and roughness. The average gap size is used to inform the proportion of gap associated to the roughness in the Form \& Waviness FEA model (see Fig. 12 with the red area associated to the average gap from the Roughness FEA being $-0.179 \mu \mathrm{m}$ ). This allows distinguishment between the gap of the form \& waviness, and roughness. Only the gap associated with the Form \& Waviness FEA model is exported at the $P_{\text {in }}$ of interest and the roughness contact is removed.

This exported model has an outlet and inlet 'buffer' added to it so that when using the CFD solver, the fluid flow converges. This allows leakage associated with only the form and waviness to be calculated. For further detailed information about how to export this using ANSYS, refer to reference [13].

\section{CFD model}

For the following CFD simulations, it is assumed that the fluid is an ideal-gas (295K) of laminar air flow solved using the Navier-Stokes equations. The wall boundary conditions (i.e. top and bottom of channel) has a low pressure boundary slip condition applied which allows the Maxwell's model for velocity slip and temperature change to be considered $\left(0.01<K_{n}<0.1\right)$ [14]. The inlet boundary condition is set to $P_{i n}$ and the outlet is set to $0 \mathrm{MPa}$.

To find the leakage of the fluid through the deformed Form \&
Waviness gap, the exported model is volume meshed using hexahedral elements (3,485,969 nodes and 3,312,000 elements) as shown in Fig. 12. The circumferential ends of the model also have a symmetric boundary condition applied. Solving this CFD model results in finding the leakage attributed to the form and waviness surface characteristics.

\section{VALIDATION}

To begin validating this new 1-way FEA-CFD numerical modelling strategy two methods are used: an analytical equation; and an experimental set-up measuring leakage of the springloaded PRV.

The analytical equation is formulated by Arkilic et al [15]. Their analytical Eqn. (2) is a modified Navier-Stokes equation which has shown very good agreement with experimental results for long microchannels $(L / h \gg 1)$.

$$
\dot{m}=\frac{w h^{3}}{24 \mu R T L}\left[P_{\text {in }}^{2}-P_{\text {out }}^{2}+12 \frac{2-\sigma}{\sigma} K_{n} P_{\text {out }}\left(P_{\text {in }}-P_{\text {out }}\right)\right]
$$

The experiment used to measure the leakage of the springloaded PRV is conducted in accordance to the API527 standard [3] and is set up as shown in Fig.13, The standard recommends to use the bubble method to assess leakage. Based on the standard, this particular valve has to leak less than 20 bubbles/min. The validation and following results are conducted for the springloaded PRV for a $P_{\text {set }}=0.5 \mathrm{MPa}$. 


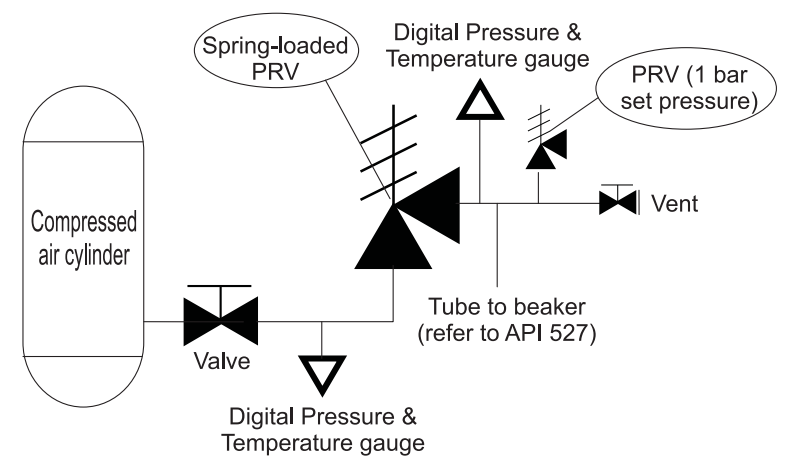

FIG. 13 SPRING-LOADED PRV EXPERIMENT SET UP IN ACCORDANCE TO API STANDARD 527

\section{RESULTS AND DISCUSSION}

\section{FEA - von Mises Stress}

Fig. 14 and 15 show the von Mises stress plot obtained across both models when the full spring force is applied at the end of the first load step.

The Form \& Waviness model (Fig. 14) rigid surface comes in contact on one-side which is expected since the profile of the form followed a sinusoidal shape. It is also noted that the model does not reach the yield stress, thus no plastic zones are present.

The Roughness model (Fig. 15) yields at the higher peak points and has a much higher stress distribution across the surface. This is expected considering the peak points would come in contact first. The plastic yielding does not extend a great deal due to the force not being high enough to do so. However, it is noted that the interaction of the asperities at the surface level have a certain influence on evolving the contact stress at the base. This evolving contact at the base has been noted in experiments conducted by Uppal and Probert [16] who studied the deformation effects on single and multiple asperities on metal surfaces.

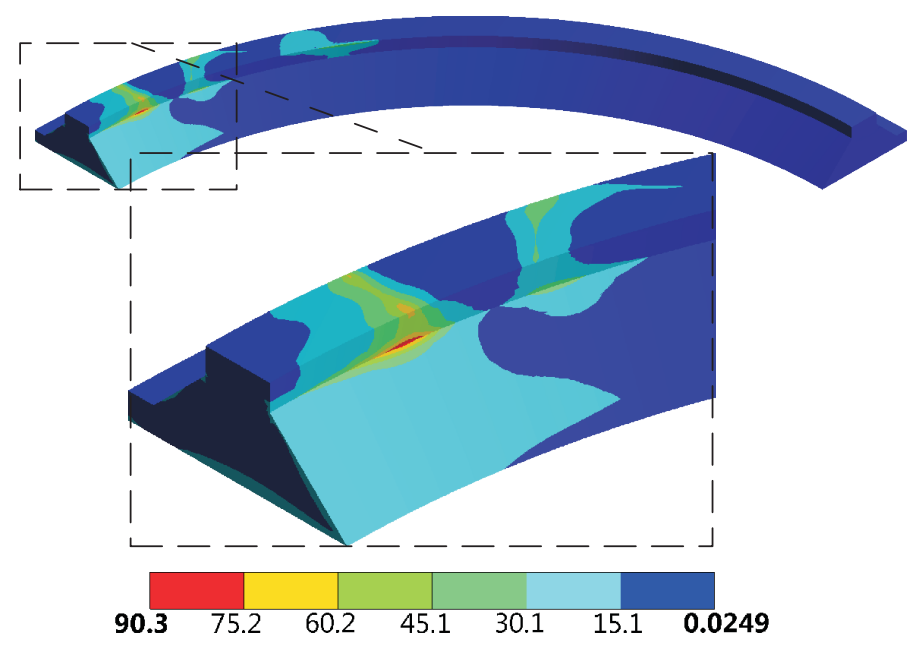

FIG. 14 VON-MISES STRESS PLOT OF FORM \& WAVINESS MODEL IN MPA

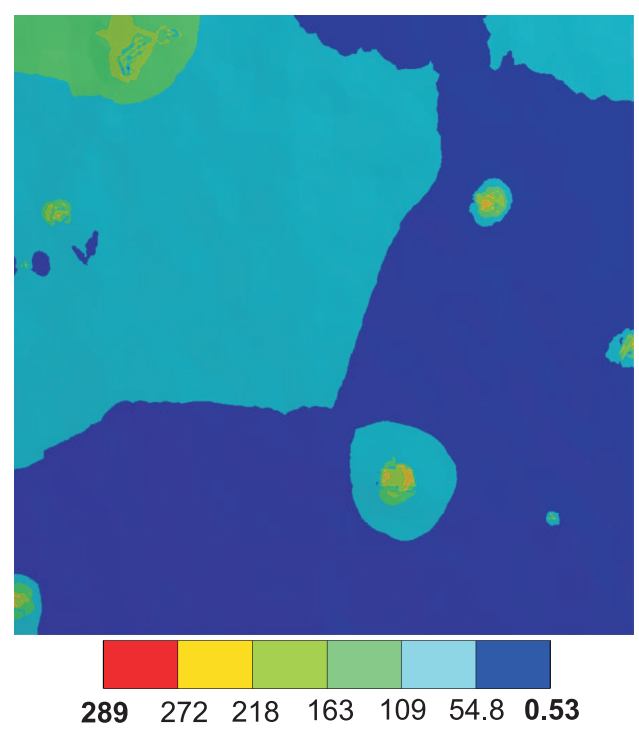

FIG. 15 VON-MISES STRESS PLOT OF ROUGHNESS MODEL IN MPA

\section{CFD - Leakage}

The valve leak tightness numerical method was solved for $P_{\text {in }}$ of $75 \%-99.5 \%$ for a $P_{\text {set }}=0.5 \mathrm{MPa}$ to allow comparison to the analytical model (Arkiclic et al) and experimental results. To allow comparison to the experimental results, which are measured in bubbles/min, the results from both the valve leak tightness numerical method and analytical model are converted to bubbles/min based on the tube radius (see Fig. 13). The results are displayed in Fig. 16. The analytical equation developed by

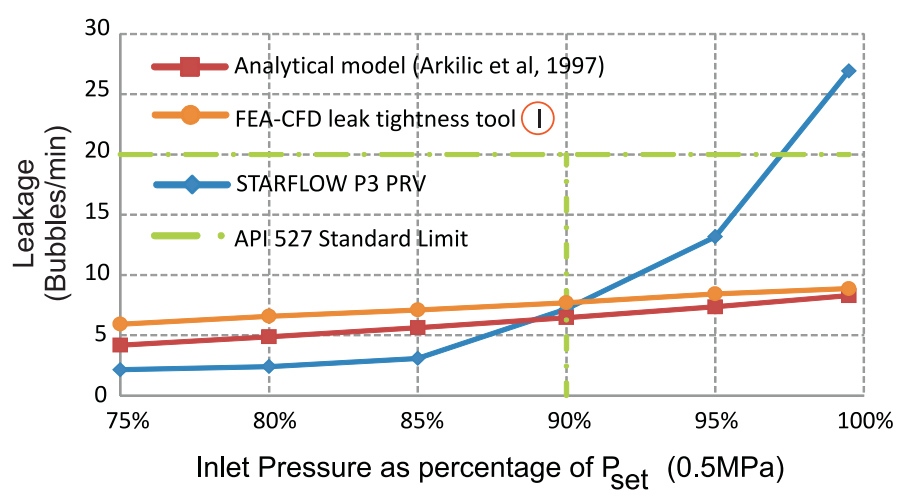

FIG. 16 LEAKAGE RESULTS OF VALVE LEAK TIGHTNESS FEA-CAD MODEL, ANALYTICAL Eqn. (2) AND PRV EXPERIMENTAL RESULTS

Arkilic shows very good correlation with the valve leak tightness numerical method. Both results show a linear trend in volumetric flow rate and a difference of $16 \%$. This difference is in-line with initial 2D validation work [17] conducted and so gives good 
confidence in the numerical FEA-CFD leak tightness modelling method.

The experimental results show an exponential change in the leakage in comparison to both the analytical and numerical linear results. Closer inspection of the experimental set-up and using a high speed camera, it was found that the bubbles which exit the tube do not hold a constant bubble radius, rather the bubble radius becomes sporadic and less spherical as we reach the $P_{\text {set }}$. However, this does not infer that the bubble volume was not consistent due to the changing bubble spherical nature.

Considering the API 527 only recommends to measure leakage at $90 \%$ of $P_{\text {set }}$, then this result alone in comparison to the numerical method shows a difference of $6.97 \%$. However, knowing that the trend in the leakage is clearly different in comparison to the results and that the bubble radius is a potential issue, the experiment needs improvement of leakage measurement.

Using Eq. (2) the average surface form \& waviness gap can be calculated based on the leakage rate obtained via the experiment at $99.5 \%$. It is found that the average gap size is $9.8 \mu \mathrm{m}$ which is greater than the measured average surface form (Fig. 4 and Table 1). There is good confidence in the metrology results since they were validated using a second optical interferometer device. Therefore, this means that the measured leakage rate is incorrect or an artefact in the experiment is not being captured in the FEA-CFD solver. Future work will look at improving how to measure and quantify leakage more accurately by using a mass spectrometer.

\section{Design optimisation - Seat Length}

The strength in this numerical tool comes from the ability to adapt the PRV geometry, such as the seat length (L) and assess the consequential leakage. Changing the seat length for a $P_{\text {set }}=0.5 \mathrm{MPa}$, it is found that by increasing the seat length by a factor of 5, leads to a reduction in leakage by $70 \%$ (see Fig. 17). Reducing the seat length increases the leakage in an inverse exponential manner, i.e. reducing the seat to half its original length results in a $60 \%$ increase in leakage.

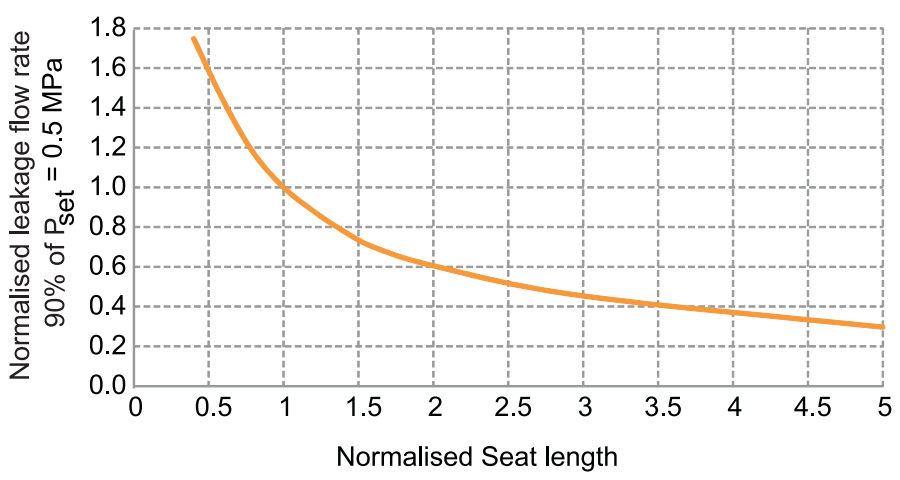

FIG. 17 SEAT LENGTH OPTIMISATION OF PRV FOR A $P_{\text {set }}=0.5 \mathrm{MPA}$

\section{Tool improvements}

For this particular study the leakage associated to the roughness was excluded since the gap created via the form and waviness was the greatest. Incorporating the roughness scan into the Form \& Waviness macro-micro CAD model would be computationally expensive.

Future efforts will focus on creating a sub-model of the sample $100 \mu \mathrm{m}$ square scan (Fig. 5) from which the leakage attributed to only the roughness could be found.

Also further validation of the summing technique will follow.

\section{Other Tool capabilities}

Based on the $P_{\text {set }}$, the loading applied in the FEA Form $\&$ Waviness model remained in the elastic regime, while the Roughness model partially began to plastically strain. However, the benefit of having a FEA-CFD solver allows for higher setpressure's to be examined, meaning that there is the potential for plastic strain to be developed across the Form \& Waviness model using a higher spring force, closing the gap height further between the metal-to-metal contacts, which in turn would reduce the leakage even further.

Other parameters such as the key metrology characteristics could be modified to find the best surface finish quality to reduce leakage, which in-turn, would be used to inform the surface finishing manufacturing quality.

Due to the 4 key connected components of the numerical model - Metrology, CAD, FEA and CFD - there individual adaptability and use in a commercial code, means this numerical model could also be utilised to analyse and improve the leakage of other metal-to-metal contact surfaces for valves, pipes and gaskets.

\section{CONCLUSIONS}

A new numerical methodology using metrology measurements - average surface form, waviness and roughness - incorporated into a 1-way FEA-CFD solver has been developed and validated for leak tightness of a PRV for a $P_{\text {set }}=0.5 \mathrm{MPa}$. The leakage result found via the numerical method in comparison to the spring loaded PRV show a disparity, however potential reasons have been identified and are being investigated. The leakage results found via the numerical method is within $16 \%$ of an analytical equation developed by Arckilic et al.

Using the numerical modelling tool the seat length was optimised showing a reduction in leakage of $70 \%$ for a $P_{\text {set }}=$ $0.5 \mathrm{MPa}$ by increasing the seat length by a factor of 5 . 


\section{ACKNOWLEDGMENT}

There are many people without whom this research project could not have been possible. The authors would like to thank:

-The individuals whose research has been mentioned in this paper, since without their great work, this work would have not come about;

-The WEIR group and the WEIR Advanced Research Center (WARC) in particular, Alan Bickley, Allan Stewart, Stéphane Carrier and Fabrice Courdavault;

-Brian Kyte (Sales Director at Alicona UK) for the help in getting access to the Alicona InfiniteFocus and addressing technical matters;

-Liza Hall (University of Strathclyde, Advanced Forming and Research Centre, Metrology) for allowing to use the Alicona InfiniteFocus and addressing technical matters;

-Rong Su and Wahyudin Syam (University of Nottingham, Institute for Advanced Manufacturing) for allowing to use the Alicona InfiniteFocus and addressing technical matters; -Ricky Martin at ARCHIE-WEST (University of Strathclyde) - Results were obtained using the EPSRC funded ARCHIE-WeSt High Performance Computer (www.archiewest.ac.uk) EPSRC grant no. EP/K000586/1.

\section{NOMENCLATURE}

$\begin{array}{ll}h & \text { channel height } \\ k & \text { Boltzmann's constant } \\ L & \text { channel length } \\ R & \text { gas constant } \\ T & \text { temperature }\end{array}$

$A_{\text {eff }} \quad$ effective Disc area

$E_{1} \& E_{2} \quad$ Young's Modulus of disc (1) and seat (2)

$E_{q} \quad$ Equivalent Young's Modulus

$F_{\text {spring }} \quad$ spring force

$F_{\text {local spring }}$ local spring force

$K_{n} \quad$ Knudsen Number

$P_{\text {in }} \quad$ inlet pressure

$P_{\text {out }} \quad$ outlet pressure

$P_{\text {set }} \quad$ set pressure

$R_{a} \quad$ average roughness

$R_{\text {in }} \quad$ inner radius of seat

$R_{\text {out }} \quad$ outer radius of seat

$R_{S m} \quad$ average roughness spacing between peaks/valleys

$W_{a} \quad$ average waviness

$W_{s m} \quad$ average waviness spacing between peaks/valleys

$\dot{m} \quad$ mass flow rate

$\mu \quad$ viscosity

$\sigma \quad$ accommodation coefficient

$\sigma_{y} \quad$ yeild stress

$v_{1} \& v_{2} \quad$ poisson ratio of disc (1) and seat (2)

\section{REFERENCES}

[1] Ralph A. Dodds, I., 2013. SRV-3B safety relief valve declared inoperable due to leakage and setpoint drift. Report no. 2013-002-00, U.S. Nuclear Regulatory Commission, Attn: Document Control Desk, Washington, D.C. 20555, Mar.

[2] Watanabe, N., 2008. "Trend analysis of incidents involving setpoint drift in safety or safety/relief valves at us lwrs". Nippon Genshiryoku Gakkai Wabun Ronbunshi, 7(1), pp. 74-84.

[3] API, 2014. Seat Tightness of Pressure Relief Valves. No. 527 in API Standard. American Petroleum Institute, Washington, USA.

[4] BSI, 2013. Safety devices for protection against excessive pressure. Safety valves. No. BS EN ISO 4126-1:2013 in British Standard. The British Standards Institution, London, UK.

[5] ASME, 2014. Pressure Relief Devices - Performance Test Codes. No. ASME PTC 25-2014 in An American National Standard. The American Society of Mechanical Engineers, New York, USA.

[6] Burmeister, L. C., Loser, J. B., and Sneegas, E. C., 1967. Advanced valve technology - Revised and enlarged edition. Technology Survey no. NASA SP-5019, Midwest Research Institute, NASA, Washington, D.C., USA.

[7] BHR Group Ltd., 2000. Valve stem leak - tightness test methodologies. Summary report no. CR1234, European Commission, British Hydromechanics Research Group Ltd., Cranfield, UK, Oct.

[8] Anwar, A. A., Gorash, Y., and Dempster, W., 2016. “Application of multi-scale approaches to the investigation of sealing surface deformation for the improvement of leak tightness in pressure relief valves". In Advanced Methods of Continuum Mechanics for Materials and Structures, K. Naumenko and M. Aßmus, eds. Springer, Singapore, pp. 493-522.

[9] O'Callaghan, P., and Probert, S., 1987. "Prediction and measurement of true areas of contact between solids". Wear, 120(1), pp. 29-49.

[10] Robbe-Valloire, F., Paffoni, B., and Progri, R., 2001. "Load transmission by elastic, elasto-plastic or fully plastic deformation of rough interface asperities". Mechanics of Materials, 33(11), pp. 617-633.

[11] Corsini, M., Cignoni, P., and Scopigno, R., 2012. "Efficient and flexible sampling with blue noise properties of triangular meshes". IEEE Transaction on Visualization and Computer Graphics, 18(6), pp. 914-924.

[12] Bernardini, F., Mittleman, J., Rushmeier, H., Silva, C., and Taubin, G., 1999. "The ball-pivoting algorithm for surface reconstruction". IEEE Transactions on Visualization and Computer Graphics, 5(4), Oct., pp. 349-359.

[13] Anwar, A. A., Gorash, Y., Dempster, W., and Nash, D., 2016. "Deformed gap space using macro-micro FEA model and transferred into a CFD model". In Proc. Joint DMV \& GAMM Annual Meeting (GAMM 2016), GAMM e.V. 
March 7-11.

[14] ANSYS ${ }^{\circledR}$ Help, 2015. Fluent // Theory Guide // 7. Species Transport and Finite-Rate Chemistry // 7.2.3. Slip Boundary Formulation for Low-Pressure Gas Systems, Academic Research 16.1.0 ed. ANSYS, Inc., Canonsburg (PA), USA.

[15] Arkilic, E. B., Schmidt, M., Breuer, K. S., et al., 1997. "Gaseous slip flow in long microchannels". J. of Microelectromechanical Systems, 6(2), pp. 167-178.

[16] Uppal, A., and Probert, S., 1972. "Deformation of single and multiple asperities on metal surfaces". Wear, 20(3), pp. 381-400.

[17] Anwar, A. A., Ritos, K., Gorash, Y., Nash, D., and Dempster, W., 2016. "Leakage of gas flow through a microchannel in the slip flow regime". In Proc. ASME Pressure Vessels \& Piping Conf. (PVP 2016), ASME. July 17-21. 